\title{
Does Mechanocrine Signaling by Liver Sinusoidal Endothelial Cells Offer New Opportunities for the Development of Anti-fibrotics?
}

\author{
Sumeyye Soydemir ${ }^{1 \dagger}$, Olivia Comella ${ }^{1 \dagger}$, Dina Abdelmottaleb ${ }^{1,2}$ and James Pritchett ${ }^{1,2 *}$ \\ ${ }^{1}$ Department of Life Sciences, Faculty of Science and Engineering, Manchester Metropolitan University, Manchester, \\ United Kingdom, ${ }^{2}$ Centre for Bioscience, Faculty of Science and Engineering, Manchester Metropolitan University, \\ Manchester, United Kingdom
}

Keywords: liver, endothelial, LSEC, PIEZO, HSC, fibrosis, ECM, mechanocrine

\section{OPEN ACCESS}

Edited by:

Shannon Glaser,

Texas A\&M Health Science Center,

College of Medicines, United States

Reviewed by:

Roberto Gramignoli,

Karolinska Institutet (KI), Sweden Anabel Fernández-Iglesias,

August Pi i Sunyer Biomedical Research Institute (IDIBAPS), Spain

*Correspondence:

James Pritchet

j.pritchett@mmu.ac.uk

†These authors have contributed equally to this work

Specialty section:

This article was submitted to

Gastroenterology,

a section of the journal

Frontiers in Medicine

Received: 08 October 2019

Accepted: 11 December 2019

Published: 09 January 2020

Citation:

Soydemir S, Comella O, Abdelmottaleb $D$ and Pritchett J (2020) Does Mechanocrine Signaling by Liver Sinusoidal Endothelial Cells Offer New Opportunities for the

Development of Anti-fibrotics?

Front. Med. 6:312.

doi: 10.3389/fmed.2019.00312

\section{INTRODUCTION}

Liver sinusoidal endothelial cells (LSECs) are specialized endothelial cells that have essential roles in normal liver homeostasis, and are also involved in disease processes. The importance of LSEC biology has recently been extensively reviewed $(1,2)$. LSECs line the walls of the hepatic sinusoid (Figure 1) where they scavenge blood borne macromolecules. LSECs are constantly exposed to antigens carried from the gastrointestinal tract by the portal vein. LSECs therefore have a crucial role, alongside Kupffer cells, as gate keepers for liver immunomodulation. If LSEC immune responses are dysregulated, the result is chronic inflammation which can drive the development of fibrosis (2).

LSECs maintain a perforated plasma membrane to form fenestrations ranging between 50 and $300 \mathrm{~nm}$ in diameter (3). In a healthy, functioning liver, blood enters the sinusoids via the portal vein and hepatic artery, thus enabling oxygen and macromolecules to be transferred across the endothelial barrier to hepatocytes, facilitated by the LSEC fenestrae (1).

Due to their location lining the sinusoid LSECs (Figure 1) are in direct contact with blood flow and therefore exposed to changes in both shear stress and blood pressure. Numerous researchers have made this observation, however recent reviews of LSEC biology $(1,2)$ also illustrate how little is known about mechano-sensing pathways in LSECs. A recent article by Hilscher et al. (4) has now highlighted how mechano-sensitive pathways in LSECs can drive recruitment of circulating blood cells to drive portal hypertension. Mechanocrine signaling by LSECs can orchestrate complex responses across cell types and tissues. This article will highlight the importance of mechano-biology in LSECs during liver disease and point out important gaps in knowledge. This exciting research topic has the potential to reveal novel targets for the development of urgently needed anti-fibrotics.

Importantly LSECs are able to modulate phenotypic changes in hepatic stellate cells (HSCs) (5-7). HSCs are responsible for the altered extracellular matrix (ECM) production characteristic of liver fibrosis (8). In the healthy liver HSCs reside in the space of disse between the endothelial (LSEC) layer and epithelial (hepatocyte) layer. In response to fibrogenic cues, including inflammatory signals from hepatocytes or LSECs, HSCs alter their phenotype to become activated myofibroblasts. Activated HSCs are proliferative, migratory, and contractile cells that secrete fibrotic ECM (9). This means that mechanically induced changes in LSECs have the potential to rapidly alter HSC phenotype and drive fibrogenesis. The fact that LSEC dysfunction precedes the 


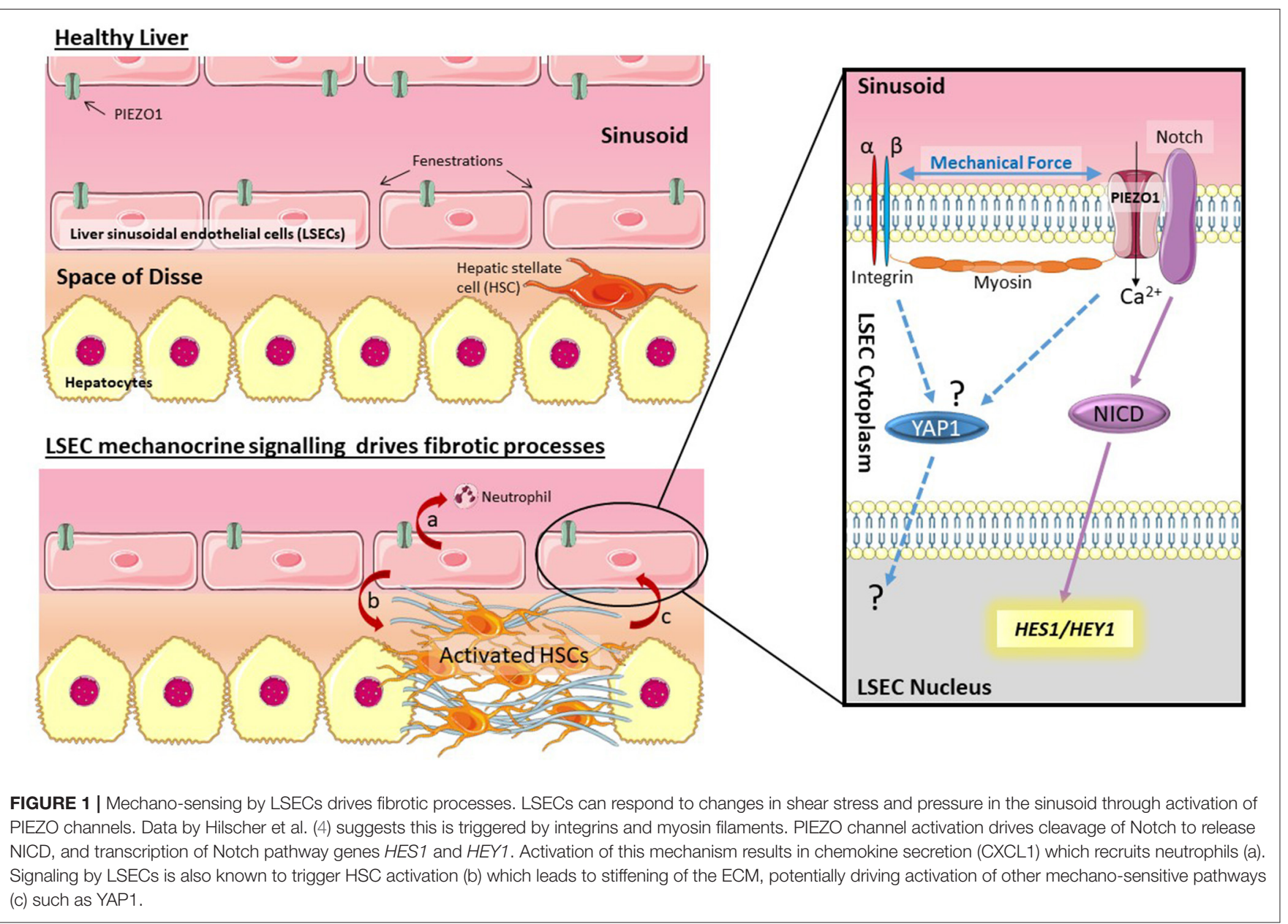

development of fibrosis in non-alcoholic liver disease (10) supports the hypothesis that signals from LSECs may be one of the earliest triggers of HSC activation. There is also the potential for the establishment of a positive feedback loop in which mechanically activated LSECs trigger mechanocrine signaling that activates HSCs. In turn, activated HSCs alter the ECM to increase tissue stiffness, driving further mechano-activation of both LSECs and HSCs. Drugs that in some way break this mechanocrine feedback loop could have great therapeutic potential for the treatment of fibrotic disease.

\section{MECHANO-BIOLOGY IN LIVER DISEASE}

Key experiments by Rebecca Wells's group clearly showed that liver stiffness changes very early following hepatic injury (11), and that increased substrate stiffness is necessary for HSC activation $(12,13)$, a key step in fibrogenesis This raises the question of whether increased hepatic stiffness is a symptom or a driver of liver disease. Or both? Mechanical force across a tissue can change due to fluctuations in blood pressure, the behavior of contractile cells (e.g., HSCs) and changes in the ECM. Following liver injury changes in hepatic blood pressure occur rapidly $(11,14)$, and hypertension in the context of non-alcoholic fatty liver disease appears to increase the risk of fibrosis $(15,16)$.
Interest in mechano-sensing during fibrotic liver disease has largely focused on HSCs $(12,17,18)$, and several mechanically sensitive signaling pathways have been shown to function in HSCs. Latent TGFbeta, a pro-fibrotic cytokine (19), is released from the ECM by contractile force transmitted from HSCs via the $\alpha_{\mathrm{v}}$ integrin subunit (20). Furthermore, the mechano-sensitive transcriptional regulator Yes Associated Protein 1 (YAP1) (21) is activated in HSCs by increased substrate stiffness $(22,23)$. YAP1 can be inhibited using verteporfin (24) to reduce fibrosis in vivo (23). By contrast, relatively little is known about how LSECs sense and respond to external mechanical cues.

\section{Portal Hypertension and Regulation of Sinusoidal Tone}

Changes in vascular tone cause rapid changes in blood pressure, shear forces and the overall mechanical stiffness of the liver (14). LSECs regulate vascular tone by releasing vasoconstrictors, e.g., cyclooxygenase 1 (COX1) and thromboxane A2 (TXA2); and vasodilators, e.g., $\mathrm{NO}$ which act on HSCs to modulate their contraction and therefore regulate sinusoidal pressure (25). Some studies suggest that endothelin, a potent vasoconstrictor, has an important role in driving portal hypertension, as patients with cirrhosis have an increased circulating ET-1 (26). When liver injury occurs, HSCs secrete Endothelin-1 (ET-1), establishing an 
autocrine loop contributing to increased blood pressure (14, 27, 28). Intriguingly, recent data suggests that ET-1 activates YAP1 in ovarian cancer cells (29). Tocci and co-workers showed that beta-arrestin, functioning downstream of $\mathrm{ET}_{\mathrm{A}} \mathrm{R}$, physically interacts with YAP1 to increase nuclear shuttling.

Research is now beginning to reveal how LSECs detect and respond to changes in hepatic blood flow and altered ECM stiffness.

\section{POTENTIAL FOR MECHANO-SIGNALING BY LSECS}

LSECs are exposed to mechanical cues derived from both blood flow/pressure changes and changes in the surrounding ECM of the liver during fibrotic disease. Endothelial cell populations in other vascular beds are able to detect and respond to mechanical cues, so it seems reasonable to suggest similar mechanisms would exist in LSECs. Several different mechanosignaling pathways, including Neurogenic locus notch homolog (Notch) 1 (30), PIEZO channels (31-33) and YAP1 (34), have all been shown to function in endothelial cells. Furthermore, as described above, ET-1 can drive YAP1 nuclear shuttling (29). This makes possible a positive feedback loop where HSCs activated by mechanical cues release ET-1, which could have a dual function. (1) Autocrine constriction of activated HSCs, contributing to portal hypertension and increased liver stiffness; and (2) YAP1 activation in both HSCs and LSECs, due to ET-1 signaling, and increased mechanical stiffness.

\section{Notch}

Notch proteins are transmembrane proteins that undergo proteolytic cleavage upon ligand binding. Notch ligands are themselves membrane bound proteins from the jagged and delta families. Upon binding to jagged or delta proteins presented by neighboring cells, Notch proteins are cleaved to release an intracellular domain (NICD) that translocates to the nucleus to orchestrate transcriptional regulation (35). This highly conserved mechanism allows cell-to-cell contact to regulate key processes such as proliferation, cell fate, differentiation, and cell death.

Notch proteins are expressed by vascular endothelial cells (36), and play a critical role in development of the vascular system (37). Mechanical force is necessary to reveal the Notch cleavage site and allow release of $\operatorname{NICD}(38,39)$. It has recently been shown that Notch 1 localization in endothelial cells is polarized by shear force. Notch1 protein polarization occurs in the direction of flow, and Notch1 is aligned with the downstream direction of flow across the endothelial cell layer (30). Furthermore, levels of nuclear NICD increased in a step wise fashion as shear stress induced by flow increased, providing compelling evidence that endothelial Notch is a mechano-sensor (30) that regulates endothelial function and phenotype in response to changes in shear stress.

In the liver Notch is expressed by LSECs $(40,41)$. Targeted deletion of Notch1, or the canonical notch effector Rbpj1, specifically in LSECs, caused dilated sinusoids and portal hypertension in adult mice (42). When Notch1 protein expression was disrupted in LSECs at birth, development of the liver vasculature was severely disrupted (42). Conversely, forced Notch pathway activation by endothelial specific overexpression of NICD also disrupted normal liver homeostasis, with expanded sinusoids, reduced hepatocyte proliferation and increased hepatocyte cell death. LSECs appeared to become dedifferentiated, and the fibrogenic response to $\mathrm{CCl} 4$ induced liver injury was increased (43).

These findings highlight the importance of tightly regulated Notch1 signaling in LSECs for normal liver function. Mechanical regulation of Notch1 could play a critical role in normal liver homeostasis, and in the response to liver injury. Intriguingly, recent data (4) shows that the Notch1 pathway in LSECs is sensitive to mechanical cues. Hilscher et al. (4) suggest that stretch activated PIEZO cation channels activate Notch signaling which drives recruitment of neutrophils and formation of neutrophil extracellular traps that cause portal hypertension.

\section{PIEZO Channels}

PIEZO proteins form mechano-sensitive cation channels in the plasma membrane $(44,45)$. PIEZO1 is essential for correct vascular development, and global knockout of PIEZO1 is lethal $(31,32)$. PIEZO1 channels are present in the plasma membrane of endothelial cells and activated by shear stress to trigger Calcium influx into the cell $(31,32)$. Since their initial discovery, it has been shown that PIEZO1 is also critical for normal vascular homeostasis. Endothelial cells respond to changes in shear forces via PIEZO1. PIEZO1 induced signaling elicits downstream changes in vascular tone and blood pressure. In mice with endothelial specific PIEZO1 deficiency the ability of endothelial cells to respond to changes in flow by releasing $\mathrm{NO}$ to trigger vasodilation was lost, resulting in hypertension (33).

PIEZO channels are present on LSECs (31), and, as mentioned above, Hilscher et al. have recently highlighted how PIEZO1 channels modulate Notch pathway activity in response to changes in blood pressure (4). In their experimental model of cyclic stretch, integrins transmitted changes in mechanical force to activate PIEZO1 cation channels, possibly via myosin (46, 47). Similarly, force transmitted via non-muscle myosin has recently been shown to be involved in the ligand-activated cleavage of Notch (48). In LSECs the integrin-activated PIEZO1 channels interact with the Notch1 receptor to activate Notch target genes via production of the transcription factors Hes1 and Heyl (4). Future experiments are necessary to establish whether myosin filaments in LSECs can interact directly with Notch1, or via PIEZO1, to drive notch cleavage and downstream signaling. It is also important to note that the actomyosin cytoskeleton has a crucial role in maintaining the fenestrated plasma membrane characteristic of healthy LSECs (49-51). This adds further complexity to the interplay between external and internal mechanical forces. How are changes in external force transmitted into LSECs? How do changes in external force affect the LSEC cytoskeleton? Could external mechanical cues have a direct influence on the maintenance of the fenestrated plasma membrane? 


\section{YAP1}

Another mechanism for mechano-signaling in LSECs is YAP1, which has recently been shown to be sensitive to shear forces in zebrafish endothelial cells (34). Nuclear YAP1 is also present in primary LSECs isolated from murine livers (52). YAP1 can be activated downstream of PIEZO1 (46). Further work is therefore necessary to confirm YAP1 expression and function in mammalian LSECs, and whether YAP1 status in LSECs can be regulated by PIEZO channel activation. Current understanding of YAP1 function in the liver has recently been extensively reviewed (53).

\section{THERAPEUTIC POTENTIAL}

LSEC phenotype restoration through inhibition of mechanosensitive pathways provides an intriguing therapeautic strategy for the treatment, and even reversal, of liver fibrosis. Compelling evidence that LSECs signal to neighboring cells in a context dependent manner to drive either tissue regeneration or fibrosis (7) provides strong support for the targeting of LSECs as a means to drive fibrosis regression. As many of the pathways discussed are not specific to LSECs, or even to endothelial cells, a means of delivering a therapy specifically to LSECs is desirable. Nano-particles targeting LSECs for the regulation of auto-immunity have already been developed (54). Similar approaches could be used to deliver molecules targeting mechano-sensing pathways specifically to LSECs. Timing of therapy will be crucial. Early intervention would arguably provide more chance of success, but this is made challenging due to issues with late diagnosis. However, clearance of hepatitis $\mathrm{C}$ infection leads to fibrosis regression, and clearly shows that human liver fibrosis is reversible at later stages than previously thought (55).

\section{Targeting Notch}

Two classes of drug that target notch signaling are currently in clinical trials as cancer therapies (56). (1) Gamma-secretase inhibitors (GSIs) target the enzymes responsible for cleavage of Notch and block release of NICD. (2) Monoclonal antibodies block notch-ligand receptor interactions. Both classes of drug have dose limiting side effects linked to normal notch function in the gastrointestinal tract. Successful adoption of notch inhibition as a therapeutic strategy for liver fibrosis would therefore require cellular targeting to avoid severe side effects. As mentioned previously (section NOTCH), Notch has diverse functions during liver development, homeostasis and disease (57). In hepatocytes (58) or LSECs (43) Notch signaling can induce HSC activation and promotes fibrosis. It has been demonstrated that inhibition of Notch signaling using a GSI in vivo ameliorated fibrosis in a CCl4 pre-clinical model (59). Therefore, therapeutic targeting of Notch would impact multiple pro-fibrotic mechanisms, potentially including mechano-crine signaling by LSECs (4).

\section{Targeting PIEZO Channels}

Yoda1 was the first molecule identified which could artificially regulate PIEZO channel activity (60). However, Yoda1 functions as an agonist and causes activation of PIEZO1. Based on the evidence from Hilscher et al. activating PIEZO1 would have a negative impact on liver fibrosis (4). Dooku is a more recently identified analog of Yoda1, which appears to function as a Yoda1 antagonist (61). Importantly this molecule only inhibits Yoda1 induced PIEZO channel activation. As yet, no small molecule antagonists of PIEZO channel mechano-activation have been discovered. It is interesting to speculate what effect PIEZO channel inhibitors might have on liver fibrosis, especially if they could be delivered specifically to LSECs. As PIEZO receptors are widely expressed across endothelial cell types, long term global treatment with a PIEZO antagonist would likely have undesirable side effects.

\section{Integrins}

Hilscher et al. demonstrate that PIEZO channel mechanoactivation is triggered by integrin signaling; treatment of cells with arginine-glycine-aspartate (RGD) peptide inhibited stretchinduced transcription of Notch target genes (4). Identification and targeting of the integrin heterodimers (62) involved in this mechanism could be a strategy for developing antifibrotics. The integrin subunits present in the LSEC cell membrane are yet to be fully characterized. Mass spectrometry showed that integrin beta 3 is expressed by LSECs following partial hepatectomy (63). Candidate integrin alpha subunits include alphaV and alphaIIb, both of which partner with the beta3 subunit to facilitate interactions between LSECs and platelets (64).

\section{Targeting YAP1?}

Verteporfin (tradename Visudyne, Novartis) was originally developed as a light activated treatment for neovascular macular degeneration (65). Verteporfin's ability to inhibit YAP1 activity was identified by screening for compounds able to disrupt the interaction between YAP-1 and it's DNA binding partner TEAD1 (24). Mice tolerate verteporfin treatment via intraperitoneal injection over 3 weeks (23). However, further studies are needed to assess its specificity and potential for development as a long term therapeutic strategy. In light of this it is important to note that more specific alternatives to verteporfin have already been developed and tested in vitro (66).

\section{DISCUSSION}

The data presented by Hilscher et al. (4) is compelling: mechanical cues alter LSEC function. In response to mechanical stretch PIEZO channels activate the notch pathway to trigger secretion of the chemokine CXCL1 by LSECs. CXCL1 release recruits neutrophils that drive microthrombi formation and promote portal hypertension. This is the first direct evidence of mechano-sensing by LSECs, and links PIEZO channels with notch-signaling, both of which are known to be mechanically activated in other contexts. It is reasonable to expect that integrins will also be involved in the detection of mechanical cues by LSECs. For other mechanosensitive pathways such as YAP/TAZ there is potential for involvement in LSEC biology 
as YAP1 responds to shear stress in a zebrafish model (34). Another area of interest is how actomyosin contractility responds to and generates force to regulate LSEC shape (fenestrae) and integrate external and internal cues via PIEZO (47), notch (48), or YAP1 (67). The next challenge will be to harness our improving understanding of the importance of mechanobiology in LSECs to attempt to develop novel therapies for liver disease. Breaking the positive feedback loop set in motion when mechanical cues cause LSECs to trigger neutrophil recruitment, and potentially HSC activation, could be a successful therapeutic strategy.

\section{AUTHOR CONTRIBUTIONS}

SS and OC researched the topic and prepared draft text and figure. DA edited the text and provided feedback. JP supervised, SS and OC managed the preparation of

\section{REFERENCES}

1. Poisson J, Lemoinne S, Boulanger C, Durand F, Moreau R, Valla D, et al. Liver sinusoidal endothelial cells: physiology and role in liver diseases. J Hepatol. (2017) 66:212-27. doi: 10.1016/j.jhep.2016.07.009

2. Shetty S, Lalor PF, Adams DH. Liver sinusoidal endothelial cells - gatekeepers of hepatic immunity. Nat Rev. (2018) 15:555-67. doi: 10.1038/s41575-018-0020-y

3. Cogger VC, McNerney GP, Nyunt T, DeLeve LD, McCourt P, Smedsrød $\mathrm{B}$, et al. Three-dimensional structured illumination microscopy of liver sinusoidal endothelial cell fenestrations. J Struct Biol. (2010) 171:382-8. doi: 10.1016/j.jsb.2010.06.001

4. Hilscher MB, Sehrawat T, Arab JP, Zeng Z, Gao J, Liu M, et al. Mechanical stretch increases expression of CXCL1 in liver sinusoidal endothelial cells to recruit neutrophils, generate sinusoidal microthombi, and promote portal hypertension. Gastroenterology. (2019) 15:193-209.e199. doi: 10.1053/j.gastro.2019. 03.013

5. Xie G, Wang X, Wang L, Atkinson RD, Kanel GC, Gaarde WA, et al. Role of differentiation of liver sinusoidal endothelial cells in progression and regression of hepatic fibrosis in rats. Gastroenterology. (2012) 14:918-27.e916. doi: 10.1053/j.gastro.2011.12.017

6. Marrone G, Russo L, Rosado E, Hide D, Garcia-Cardena G, GarciaPagan JC, et al. The transcription factor KLF2 mediates hepatic endothelial protection and paracrine endothelial-stellate cell deactivation induced by statins. J Hepatol. (2013) 5:98-103. doi: 10.1016/j.jhep.2012. 08.026

7. Ding BS, Cao Z, Lis R, Nolan DJ, Guo P, Simons M, et al. Divergent angiocrine signals from vascular niche balance liver regeneration and fibrosis. Nature. (2014) 50:97-102. doi: 10.1038/nature12681

8. Tsuchida T, Friedman SL. Mechanisms of hepatic stellate cell activation. Nat Rev. (2017) 14:397-411. doi: 10.1038/nrgastro.2017.38

9. Hernandez-Gea V, Friedman SL. Pathogenesis of liver fibrosis. Annu. Rev. Pathol. (2011) 6:425-56. doi: 10.1146/annurev-pathol-011110-130246

10. Pasarín M, La Mura V, Gracia-Sancho J, García-Calderó H, Rodríguez-Vilarrupla A, García-Pagán JC, et al. Sinusoidal endothelial dysfunction precedes inflammation and fibrosis in a model of NAFLD. PLoS ONE. (2012) 7:e32785. doi: 10.1371/journal.pone.00 32785

11. Georges PC, Hui JJ, Gombos Z, McCormick ME, Wang AY, Uemura M, et al. Increased stiffness of the rat liver precedes matrix deposition: implications for fibrosis. Am J Physiol Gastrointest. Liver Physiol. (2007) 293:G1147-54. doi: 10.1152/ajpgi.00032.2007 the manuscript, researched the topic, and prepared the final text.

\section{FUNDING}

SS received a Wellcome Trust Vacation Studentship $(218402 / Z / 19 / Z)$. We thank the Center for Bioscience at Manchester Metropolitan University for funding to support DA (MMU Strategic Opportunities Fund).

\section{ACKNOWLEDGMENTS}

We acknowledge the support of the Center for Bioscience and Department of Life Sciences at Manchester Metropolitan University. We used The SMART Medical Art platform (https://smart.servier.com/) for figure design.

12. Wells RG. The role of matrix stiffness in hepatic stellate cell activation and liver fibrosis. J Clin Gastroenterol. (2005) 3:S158-61. doi: 10.1097/01.mcg.0000155516.02468.0f

13. Olsen AL, Bloomer SA, Chan EP, Gaca MDA, Georges PC, Sackey B, et al. Hepatic stellate cells require a stiff environment for myofibroblastic differentiation. Am J Physiol Gastrointest Liver Physiol. (2011) 30:G110-18. doi: 10.1152/ajpgi.0041 2.2010

14. Rockey DC. Hepatic blood flow regulation by stellate cells in normal and injured liver. Semin Liver Dis. (2001) 2:337-49. doi: 10.1055/s-2001-17551

15. Dixon JB, Bhathal PS, O'Brien PE. Nonalcoholic fatty liver disease: predictors of nonalcoholic steatohepatitis and liver fibrosis in the severely obese. Gastroenterology. (2001) 12:91-100. doi: 10.1053/gast.2001.25540

16. Arima S, Uto H, Ibusuki R, Kumamoto R, Tanoue S, Mawatari S, et al. Hypertension exacerbates liver injury and hepatic fibrosis induced by a choline-deficient L-amino acid-defined diet in rats. Int J Mol Med. (2014) 3:68-76. doi: 10.3892/ijmm.2013.1544

17. Wells RG. Tissue mechanics and fibrosis. Biochim Biophys Acta. (2013) 183:884-90. doi: 10.1016/j.bbadis.2013.02.007

18. Daniel JT, Giovanni L, Moira BH, Vijay HS. Mechanosensing and fibrosis. J. Clin. Invest. (2018) 12:74-84. doi: 10.1172/JCI93561

19. Gressner AM, Weiskirchen R, Breitkopf K, Dooley S. Roles of TGF-beta in hepatic fibrosis. Front Biosci. 7:D793-807. doi: 10.2741/A812

20. Henderson N, Arnold T, Katamura Y, Giacomini M, Rodriguez J, McCarty J, et al. Targeting of $\alpha \mathrm{v}$ integrin identifies a core molecular pathway that regulates fibrosis in several organs. Nat Med. (2013) 19:1617-24. doi: 10.1038/nm.3282

21. Dupont S, Morsut L, Aragona M, Enzo E, Giulitti S, Cordenonsi M, et al. Role of YAP/TAZ in mechanotransduction. Nature. (2011) 47:179-83. doi: $10.1038 /$ nature 10137

22. Mannaerts I, Leite SB, Verhulst S, Claerhout S, Eysackers N, Thoen LFR, et al. The Hippo pathway effector YAP controls mouse hepatic stellate cell activation. J Hepatol. (2015) 6:679-88. doi: 10.1016/j.jhep.2015. 04.011

23. Martin K, Pritchett J, Llewellyn J, Mullan AF, Athwal VS, Dobie R, et al. PAK proteins and YAP-1 signalling downstream of integrin beta1 in myofibroblasts promote liver fibrosis. Nat Commun. (2016) 7:12502. doi: $10.1038 /$ ncomms 12502

24. Liu-Chittenden Y, Huang B, Shim JS, Chen Q, Lee SJ, Anders RA, et al. Genetic and pharmacological disruption of the TEAD-YAP complex suppresses the oncogenic activity of YAP. Genes Dev. (2012) 26:1300-5. doi: $10.1101 / \operatorname{gad} .192856 .112$

25. Gracia-Sancho J, Marrone G, Fernández-Iglesias A. Hepatic microcirculation and mechanisms of portal hypertension. Nat Rev 
Gastroenterol Hepatol. (2019) 16:221-34. doi: 10.1038/s41575-01 8-0097-3

26. Trevisani F, Colantoni A, Gerbes AL, Gülberg V, Sica G, Caraceni $\mathrm{P}$, et al. Daily profile of plasma endothelin-1 and-3 in preascitic cirrhosis: relationships with the arterial pressure and renal function. J Hepatol. (1997) 26:808-15. doi: 10.1016/S0168-8278(97)8 0246-2

27. Gandhi CR, Sproat LA, Subbotin VM. Increased hepatic endothelin-1 levels and endothelin receptor density in cirrhotic rats. Life Sci. (1996) 58:55-62. doi: 10.1016/0024-3205(95)02255-4

28. Cho TJ, Kim HJ, Cho J. Endothelin-converting enzyme-1 expression in acute and chronic liver injury in fibrogenesis. Anim Cells Syst. (2019) 23:170-5. doi: 10.1080/19768354.2019.1595141

29. Tocci P, Cianfrocca R, Di Castro V, Rosano L, Sacconi A, Donzelli S, et al. $\beta$ arrestin1/YAP/mutant p53 complexes orchestrate the endothelin A receptor signaling in high-grade serous ovarian cancer. Nat Commun. (2019) 10:3196. doi: 10.1038/s41467-019-11045-8

30. Mack JJ, Mosqueiro TS, Archer BJ, Jones WM, Sunshine H, Faas GC, et al. NOTCH1 is a mechanosensor in adult arteries. Nat Commun. (2017) 8:1620. doi: 10.1038/s41467-017-01741-8

31. Li J, Hou B, Tumova S, Muraki K, Bruns A, Ludlow MJ, et al. Piezo1 integration of vascular architecture with physiological force. Nature. (2014) 515:279-82. doi: 10.1038/nature13701

32. Ranade SS, Qiu Z, Woo SH, Hur SS, Murthy SE, Cahalan SM, et al. Piezo1, a mechanically activated ion channel, is required for vascular development in mice. Proc Natl Acad Sci USA. (2014) 111:10347-52. doi: 10.1073/pnas.1409233111

33. Wang S, Chennupati R, Kaur H, Iring A, Wettschureck N, Offermanns S. Endothelial cation channel PIEZO1 controls blood pressure by mediating flow-induced ATP release. J Clin Invest. (2016) 126:4527-36. doi: $10.1172 /$ JCI87343

34. Nakajima H, Yamamoto K, Agarwala S, Terai K, Fukui H, Fukuhara S, et al. Flow-Dependent endothelial YAP regulation contributes to vessel maintenance. DevCell. (2017) 40:523-36.e6. doi: 10.1016/j.devcel.2017. 02.019

35. Kopan R. Notch signaling. Cold Spring Harb Perspect Biol. (2012) 4:a011213. doi: 10.1101/cshperspect.a011213

36. Del Amo FF, Smith DE, Swiatek PJ, Gendron-Maguire M, Greenspan RJ, McMahon AP, et al. Expression pattern of Motch, a mouse homolog of Drosophila Notch, suggests an important role in early postimplantation mouse development. Development. (1992) 115:7 37-44.

37. Krebs LT, Xue Y, Norton CR, Shutter JR, Maguire M, Sundberg JP, et al. Notch signaling is essential for vascular morphogenesis in mice. Genes Dev. (2000) 14:1343-52. doi: 10.1101/gad.14.11.1343

38. Gordon WR, Vardar-Ulu D, Histen G, Sanchez-Irizarry C, Aster JC, Blacklow SC. Structural basis for autoinhibition of Notch. Nat Struct Mol Biol. (2007) 14:295-300. doi: 10.1038/nsmb1227

39. Wang $X, H a T$. Defining single molecular forces required to activate integrin and Notch signaling. Science. (2013) 340:991-4. doi: 10.1126/science.1231041

40. Loomes KM, Taichman DB, Glover CL, Williams PT, Markowitz JE, Piccoli DA, et al. Characterization of Notch receptor expression in the developing mammalian heart and liver. Am J Med Genet. (2002) 112:181-9. doi: 10.1002/ajmg.10592

41. Köhler C, Bell AW, Bowen WC, Monga SP, Fleig W, Michalopoulos GK. Expression of Notch-1 and its ligand Jagged-1 in rat liver during liver regeneration. Hepatology. (2004) 39:1056-65. doi: 10.1002/hep. 20156

42. Cuervo H, Nielsen CM, SimonettoDA, Ferrell L, Shah VH, Wang RA. Endothelial Notch signaling is essential to prevent hepatic vascular malformations in mice. Hepatology. (2016) 64:1302-16. doi: 10.1002/hep.28713

43. Duan JL, Ruan B, Yan XC, Liang L, Song P, Yang ZY, et al. Endothelial Notch activation reshapes the angiocrine of sinusoidal endothelia to aggravate liver fibrosis and blunt regeneration in mice. Hepatology. (2018) 68:677-90. doi: 10.1002/hep.29834

44. Coste B, Mathur J, Schmidt M, Earley TJ, Ranade S, Petrus MJ, et al. Piezo1 and Piezo2 are essential components of distinct mechanically activated cation channels. Science. (2010) 330:55-60. doi: 10.1126/science.11 93270

45. Coste B, Xiao B, Santos JS, Syeda R, Grandl J, Spencer KS, et al. Piezo proteins are pore-forming subunits of mechanically activated channels. Nature. (2012) 483:176-81. doi: 10.1038/nature 10812

46. Pathak MM, Nourse JL, Tran T, Hwe J, Arulmoli J, Le DTT, et al. Stretch-activated ion channel Piezol directs lineage choice in human neural stem cells. Proc. Natl. Acad. Sci. USA. (2014) 111:16148-53. doi: 10.1073/pnas.1409802111

47. Quintanilla MA, Hammer JA, Beach JR. Pulling in new directions: Myosin 2, Piezo, and metabolism [version 1; peer review: 3 approved]. F1000Res. (2019) 8:1486. doi: 10.12688/f1000research.18856.1

48. Hunter GL, He L, Perrimon N, Charras G, Giniger E, Baum B. A role for actomyosin contractility in Notch signaling. BMC Biol. (2019) 17:12. doi: 10.1186/s12915-019-0625-9

49. Yokomori H, Yoshimura K, Funakoshi S, Nagai T, Fujimaki K, Nomura M, et al. Rho modulates hepatic sinusoidal endothelial fenestrae via regulation of the actin cytoskeleton in rat endothelial cells. Lab Invest. (2004) 84:857-64. doi: 10.1038/labinvest.3700114

50. Yokomori H. New insights into the dynamics of sinusoidal endothelial fenestrae in liver sinusoidal endothelial cells. Med Mol Morphol. (2008) 41:1-4. doi: 10.1007/s00795-007-0390-7

51. Venkatraman L, Tucker-Kellogg L. The CD47-binding peptide of thrombospondin-1 induces defenestration of liver sinusoidal endothelial cells. Liver Int. (2013) 33:1386-97. doi: 10.1111/liv.12231

52. Zhang C, Bian M, Chen X, Jin H, Zhao S, Yang X, et al. Oroxylin A prevents angiogenesis of LSECs in liver fibrosis via inhibition of YAP/HIF- $1 \alpha$ signaling. J Cell Biochem. (2018) 119:2258-68. doi: 10.1002/jcb.26388

53. Manmadhan S, Ehmer U. Hippo signaling in the liver - a long and ever-expanding story. Front Cell Dev Biol. (2019) 7:33. doi: 10.3389/fcell.2019.00033

54. Carambia A, Freund B, Schwinge D, Bruns OT, Salmen SC, Ittrich $\mathrm{H}$, et al. Nanoparticle-based autoantigen delivery to Treg-inducing liver sinusoidal endothelial cells enables control of autoimmunity in mice. J Hepatol. (2015) 62:1349-56. doi: 10.1016/j.jhep.2015. 01.006

55. van der Meer AJ, Berenguer M. Reversion of disease manifestations after HCV eradication. J Hepatol. (2016) 65:S95-108. doi: 10.1016/j.jhep.2016.07.039

56. Venkatesh V, Nataraj R, Thangaraj GS, Karthikeyan M, Gnanasekaran A, Kaginelli SB, et al. Targeting Notch signalling pathway of cancer stem cells. Stem Cell Investig. (2018) 5:5. doi: 10.21037/sci.2018.02.02

57. Adams JM, Jafar-Nejad H. The roles of Notch signaling in liver development and disease. Biomolecules. (2019) 9:E608. doi: 10.3390/biom9100608

58. Zhu C, Kim K, Wang X, Bartolome A, Salomao M, Dongiovanni $\mathrm{P}$, et al. Hepatocyte Notch activation induces liver fibrosis in nonalcoholic steatohepatitis. Sci Transl Med. (2018) 10:eaat0344. doi: 10.1126/scitranslmed.aat0344

59. Chen Y, Zheng S, Qi D, Zheng S, Guo J, Zhang S, et al. Inhibition of Notch signaling by a $\gamma$-secretase inhibitor attenuates hepatic fibrosis in rats. PLoS ONE. (2012) 7:e46512. doi: 10.1371/journal.pone.0046512

60. Syeda R, Xu J, Dubin AE, Coste B, Mathur J, Huynh T, et al. Chemical activation of the mechanotransduction channel Piezo1. Elife. (2015) 4:e07369. doi: 10.7554/eLife.07369.008

61. Evans EL, Cuthbertson K, Endesh N, Rode B, Blythe NM, Hyman AJ, et al. Yodal analogue (Dooku1) which antagonizes Yodal-evoked activation of Piezol and aortic relaxation. Br J Pharmacol. (2018) 175:1744-59. doi: 10.1111/bph.14188

62. Raab-Westphal S, Marshall JF, Goodman SL. Integrins as therapeutic targets: successes and cancers. Cancers. (2017) 9:E110. doi: 10.3390/cancers9090110

63. Li X, Xiong L, Xie C, Cao J, Deng H, Lin Y, et al. Proteomics analysis of plasma membrane from liver sinusoidal endothelial cells after partial hepatectomy by an improved two-dimensional electrophoresis. Mol Cell Biochem. (2010) 344:137-50. doi: 10.1007/s11010-01 0-0537-z

64. Lalor PF, Herbert J, Bicknell R, Adams DH. Hepatic sinusoidal endothelium avidly binds platelets in an integrin-dependent manner, leading to platelet and endothelial activation and leukocyte recruitment. Am J Physiol. (2013) 304:G469-78. doi: 10.1152/ajpgi.00407.2012 
65. Michels S, Schmidt-Erfurth U. Photodynamic therapy with verteporfin: a new treatment in ophthalmology. Semin Ophthalmol. (2001) 16:201-6. doi: 10.1076/soph.16.4.201.10298

66. Smith SA, Sessions RB, Shoemark DK, Williams C, Ebrahimighaei R, McNeill $\mathrm{MC}$, et al. Antiproliferative and antimigratory effects of a novel YAP-TEAD interaction inhibitor identified using in silico molecular docking. J Med Chem. (2019) 62:1291-305. doi: 10.1021/acs.jmedchem.8b01402

67. Mana-Capelli S, Paramasivam M, Dutta S, McCollum D. Angiomotins link F-actin architecture to Hippo pathway signaling. Mol Biol Cell. (2014) 25:1676-85. doi: $10.1091 / \mathrm{mbc} . e 13-1$ $1-0701$
Conflict of Interest: The authors declare that the research was conducted in the absence of any commercial or financial relationships that could be construed as a potential conflict of interest.

Copyright (c) 2020 Soydemir, Comella, Abdelmottaleb and Pritchett. This is an openaccess article distributed under the terms of the Creative Commons Attribution License (CC BY). The use, distribution or reproduction in other forums is permitted, provided the original author(s) and the copyright owner(s) are credited and that the original publication in this journal is cited, in accordance with accepted academic practice. No use, distribution or reproduction is permitted which does not comply with these terms. 